\title{
Probe Correction Method
}

National Cancer Institute

\section{Source}

National Cancer Institute. Probe Correction Method. NCI Thesaurus. Code C64230.

A method for correcting perfect match (PM) probe expression levels. Defined in the documents available at http://bioconductor.org/packages/1.9/bioc/html/affy.html 